\title{
Coronary arterial origins in transposition of the great arteries: factors that affect outcome. A morphological and clinical study
}

\author{
J Li, R M R Tulloh, A Cook, M Schneider, S Y Ho, R H Anderson
}

\begin{abstract}
Objective-Transfer of the coronary arteries is crucial during the arterial switch operation for transposition, but little attention has been paid to the position of their orifices relative to the valvar sinuses. The objective of this study was to determine the factors which are important for effective transfer and to determine potential surgical significance.

Design-Morphological and clinical study.

Setting-Two national centres for neonatal cardiac surgery.

Patients-277 patients with transposition of the great arteries. One group comprised 88 necropsy specimens (ages ranging from 17 weeks of fetal life to 17 years old), and the other comprised 189 children undergoing surgery. The coronary artery orifices were inspected relative to the depth of the aortic sinuses (vertical origin), relative to the commissures between the valvar leaflets (radial origin), and their angle of exit from the aortic wall (angle of origin). The data were compared with the surgical results.

Results-In the necropsy specimens, the vertical origin of the arteries was at, or above, the sinutubular junction in $20 \%$, the radial origin was paracommissural in $3 \%$, and the angle of origin was not orthogonal in $7 \%$. Those with high take off and paracommissural origin were all intramural. In the clinical cases, those children with high take off, paracommissural origin or tangential origin had an increased risk at surgery.

Conclusions-In $20 \%$ of hearts, high take off, paracommissural orifice, or tangential origin of coronary arteries is found. This may be recognised preoperatively by echocardiography and may cause technical difficulty in transfer during the arterial switch procedure.
\end{abstract}

(Heart 2000;83:320-325)

Keywords: transposition of the great arteries; coronary arteries; arterial switch operation; echocardiography; anatomy; congenital heart defects

\section{Department of \\ Paediatrics, Imperial \\ College School of \\ Medicine at National \\ Heart and Lung \\ Institute, London, UK \\ $\mathrm{J} \mathrm{Li}$ \\ $S$ Y Ho \\ R H Anderson}

Department of Paediatric Cardiology, Guy's and St Thomas'

Hospital Trust, 11th

Floor Guy's Tower,

Guy's Hospital, St

Thomas' Street,

London SE1 9RT, UK

R M R Tulloh

A Cook

M Schneider

Correspondence to: Dr Tulloh

email: robert.tulloh@

GSTT.sthames.nhs.uk

Accepted 29 October 1999
The arterial switch operation is now accepted as the treatment of choice for surgical correction of hearts with concordant atrioventricular and discordant ventriculo-arterial connections (transposition of the great arteries (TGA)). ${ }^{1-6}$ The overall mortality is now low, but the branching patterns of the coronary arteries are known to be variable and to influence outcome. Some patterns have been associated with mortality probability of up to $30 \% .^{12}$ Occasionally, one or both coronary arteries may run in the wall of the aorta (so called intramural origin), usually increasing the surgical risk, ${ }^{1}{ }^{25-7}$ although some surgeons describe little effect on outcome. ${ }^{8}$ Such intramural coronary arteries have been studied in detail. ${ }^{125-11}$ Little attention has otherwise been paid to the origins of the coronary arteries, such as the location of the orifice in radial and vertical planes relative to the arterial sinus, and the orientation of its proximal segment, factors established as being of clinical importance in the otherwise normal heart. ${ }^{11}$

Many systems have been suggested to classify the arrangement of the coronary arteries, but some have stated that some branching patterns are not classifiable (de Leval, personal communication, 1998). We hope that the consistent and infinitely variable system suggested in our paper avoids the limitations associated with an alphanumeric system. We have studied aspects of variability of origin and assessed their importance in 277 children with TGA, to reduce the risk of the arterial switch operation.

\section{Methods}

NECROPSY SERIES

Eighty eight formalin fixed specimens with TGA were held in the cardiopathological museums of Guy's Hospital and Royal Brompton Hospital (38 male, 19 female, 31 unknown). The median age at death was 4 weeks (range 17 weeks of fetal life to 17 years old). Forty six had had no surgery, while 42 had undergone Mustard/Senning operation (16), or arterial switch (26). The ventricular septum was intact in $42(48 \%)$, whereas $46(52 \%)$ hearts had well recognised complex associations.

The origins of the coronary arteries were identified from the luminal aspect of the aorta and were defined as seen by an observer standing, figuratively, in the pulmonary trunk facing the aorta, with the left hand pointing to the left sided sinus and the right hand pointing to the right sided sinus. The left hand and right hand sinuses then correspond to sinus 1 and 2, respectively, in the Leiden convention. ${ }^{12}$ In each specimen, we recorded the number, position, and shape of the coronary artery orifices. The vertical position of each orifice was measured relative to the distance from the base of the supporting aortic sinus to the sinutubular junction, and was described as being above, 
below, or at the level of the junction. This information was represented as the ratio of the measured distance to the depth of the sinus. The radial position was measured as the distance from the orifice to the zone of apposition between the facing aortic valvar leaflets. This was represented as the ratio of this distance compared to the width of the aortic sinus at the sinutubular junction.

The orientation of each proximal coronary artery segment was noted in relation to the location of its orifice. If the orifice was at, or above, the sinutubular junction ("high take off'), or paracommissural $(<20 \%$ of the width of the sinus at the sinutubular junction), its origin and proximal segment were sectioned to determine the presence or absence of an intra-

Coronary arterial orifices in complete transposition

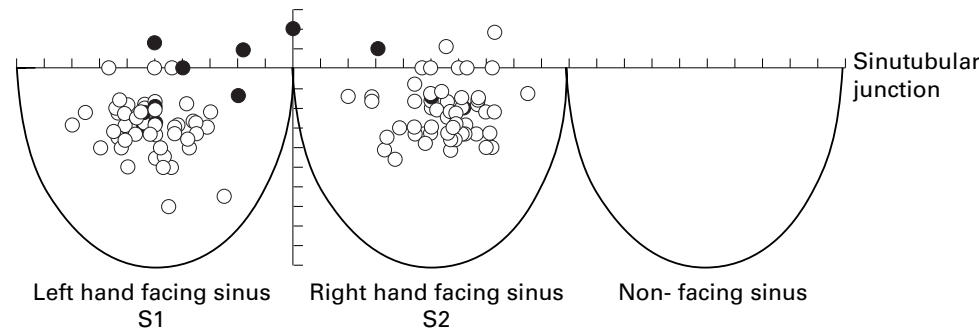

Figure 1 Vertical origin of the coronary artery orifices in the 62 specimens. The aortic root has been cut open and is represented as a flat plane. Open circles represent orthogonal origin of the coronary arteries and closed circles represent tangential or intramural coronary artery origins which would be more difficult to translocate during an arterial switch operation.

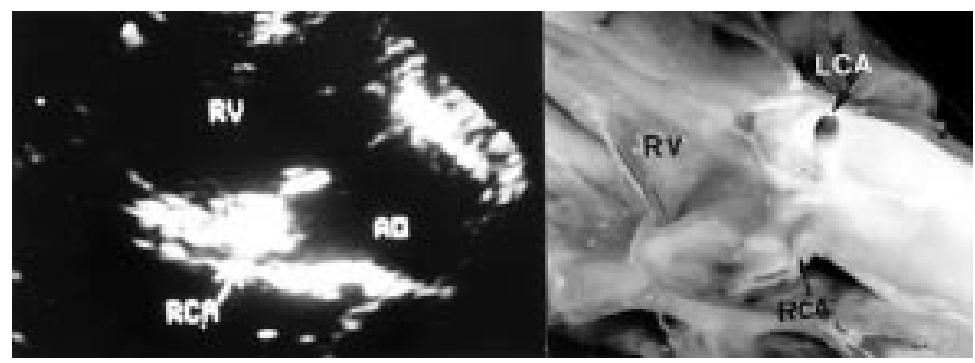

Figure 2 Representation of a right coronary artery with an orthogonal origin as seen in (left) an echocardiogram, and (right) a specimen photograph. The origin lies well within the aortic sinus and the echocardiographic case underwent successful switch operation with no complications. RCA, right coronary artery; LCA, left coronary artery; RV, right ventricle; $A O$, aorta.
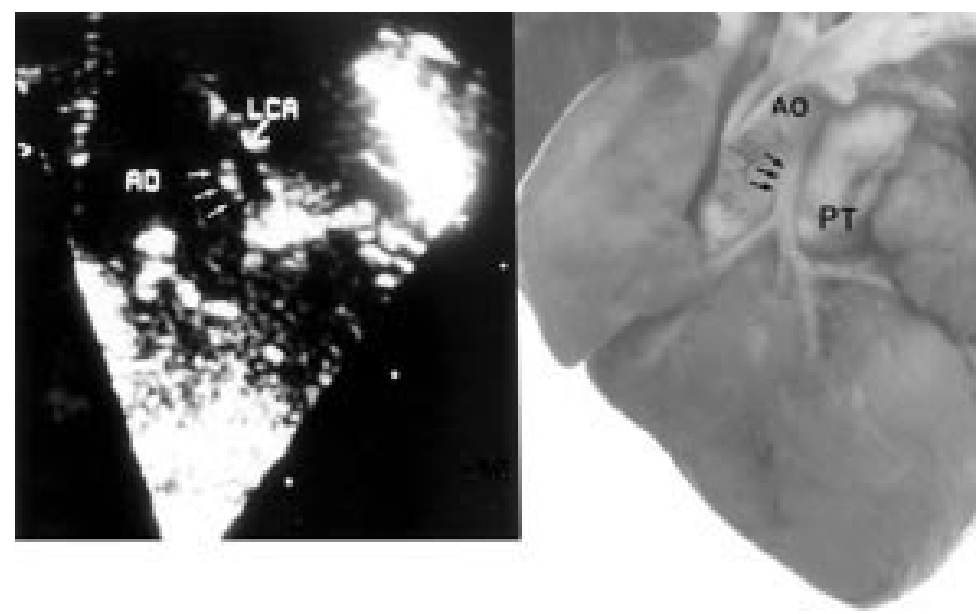

Figure 3 View of a left coronary artery origin which is above the sinutubular junction and is tangential to the aortic wall as seen in (left) an echocardiogram, and (right) a specimen photograph. The coronary artery shown in the echocardiogram was intramural but relocation was possible during the arterial switch operation. mural course. Three patterns were described: orthogonal (or nearly so) to the wall of the aorta; tangential to the aortic wall, but not intramural; and intramural coronary arteries. ${ }^{11}$ The presence of orificial stenosis was also noted.

All specimens were examined before removing perivascular fatty tissue. The coronary artery pattern was described as "usual" when the left main stem arose from the left hand sinus, and the right from the right hand sinus. Other variations were described rather than using alphanumeric classifications. ${ }^{1314}$ The orientation of the aorta and pulmonary trunk, and the origin and course of the sinus nodal artery, were noted.

SURGICAL SERIES

The echocardiograms and surgical reports of 189 patients (129 male, 60 female) were reviewed; most recorded prospectively, the others only being included if the data from the echocardiograms or surgery were sufficient. Of these children, 176 had undergone arterial switch at Guy's Hospital (84) or at the Royal Brompton Hospital (92) between January 1992 and September 1996, or other operations (Mustard (5), Rastelli (2), shunt (5), or none (1)). The median age at echocardiography was 11 days with the ventricular septum intact, or nearly so, in 131 cases $(69 \%)$ and more complex defects in 58 cases $(31 \%)$.

The vertical and radial origin, the angle of origin of the orifices (as described above), and the branching pattern of the coronary arteries were noted. If intramural, the course was noted on echocardiography, and confirmed at surgery. The echocardiographic criteria for an intramural course included "double border" appearance as the vessel wall ran parallel to the aortic wall within the adventitial covering, ${ }^{10}$ or a "high take off" or paracommissural orifice, giving rise to an acute tangential angle of origin. These observations were correlated with the surgical outcome.

\section{Results}

MORPHOLOGIC FINDINGS

Coronary arterial orifices

We examined 62 specimens from patients who had not undergone the arterial switch procedure (fig 1).

In those arising from the left hand sinus, the orifice was below the junction in $53(87 \%)$, at the junction in five $(8 \%)$, and above in three ( $5 \%$ ) (figs 2,3 , and 4 ). In one, there was no coronary artery arising from this sinus. The mean (SD) vertical origin was 26.5 (17)\% below the junction, ranging from $70 \%$ below to $30 \%$ above. In two, the orifice of infundibular branches arose, one at $30 \%$ above, and in the other below the junction. One orifice was located $20 \%$ above the commissure between the facing sinuses, giving rise to an intramural accessory left anterior descending coronary artery.

The mean radial origin was between $0-80 \%$ from the zone of apposition of the facing commissures. Paracommissural origins were found 


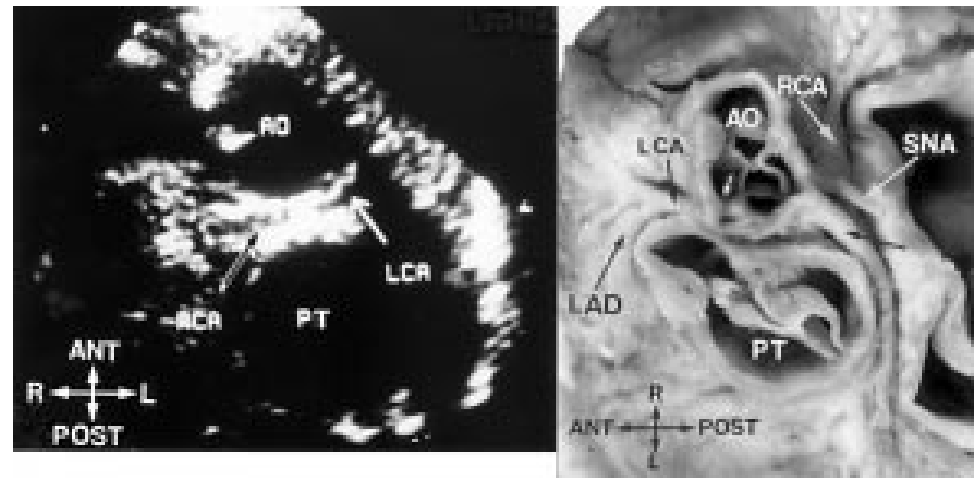

Figure 4 View of single origin of the coronary arteries from the right facing sinus with the left coursing between the aorta and pulmonary artery as seen in (left) an echocardiogram, and (right) a specimen photograph. Coronary artery relocation was not possible, the aorta being reconstructed so as to redirect flow into the coronary artery following the switch operation, without relocation. LAD, left anterior descending artery; PT, pulmonary trunk; SNA, sinus nodal artery.

in two hearts. Two orifices, separated by a distance of $3.5 \mathrm{~mm}$, were found in one specimen, giving rise to the left and intramural right coronary arteries.

The angle of origin was nearly orthogonal in both vertical and horizontal axes in the majority of cases (53/61). The origin was tangential, but without an obvious intramural segment, in four $(6 \%)$ specimens, arising at or above the sinutubular junction and in each case having a paracommissural orifice. The artery coursed intramurally in four further cases. In one further case, the left coronary artery descended on the adventitial surface of the aorta before passing round the pulmonary trunk. Although not intramural, it would have been difficult to separate the artery from the aorta. One orifice was stenosed, being $50 \%$ smaller than the internal diameter of the distal artery.

For the arteries originating from the right hand sinus, the orifice was below the sinutubular junction in 52 specimens (87\%), at the level in five $(8 \%)$, and above in three $(5 \%)$. In two hearts, there was no coronary artery arising from this sinus. The mean vertical origin was $22(14) \%$ below the junction, ranging from $46 \%$ below to $14 \%$ above. The mean radial origin was 55 (11)\% (range 29-88\%) from the facing commissures with only one having a paracommissural orifice. There were two orifices in the right hand sinus in four specimens, less than $2 \mathrm{~mm}$ from each other. They gave rise to right and circumflex coronary arteries in two specimens, and to the right and

Table 1 Distribution of coronary artery branching patterns and presence of intramural coronary arteries. Correlation with outcome

\begin{tabular}{llccl}
\hline Sinus of origin & Specimens & $\begin{array}{l}\text { Patients } \\
(n(\%))\end{array}$ & Comments \\
\hline Sinus 1 & Sinus 2 & $57(65)$ & $122(65)$ & 5 intramural/6 deaths \\
\hline L Cx & R & $14(16)$ & $35(18)$ & 1 intramural $/ 5$ deaths \\
L & R Cx & $4(5)$ & $4(2)$ & 1 intramural $/ 2$ death \\
& L R Cx & $1(1)$ & $4(2)$ & 1 intramural $/ 1$ death \\
L R Cx & L R Cx & $2(2)$ & $2(1)$ & 1 intramural \\
L & L R & $0(0)$ & $2(1)$ & 1 intramural \\
L Cx & Cx & $4(5)$ & $13(7)$ & 1 intramural $/ 4$ deaths \\
L R & L Cx & $5(6)$ & $4(2)$ & 2 deaths \\
R & L R & $1(1)$ & $3(2)$ & \\
Cx & & $88(100)$ & $189(100)$ & 11 intramural $/ 20$ deaths \\
Total & & &
\end{tabular}

$\mathrm{L}$, left coronary artery; $\mathrm{R}$, right coronary artery; $\mathrm{Cx}$, circumflex coronary artery. left coronary arteries in the others. No other accessory orifices were found.

The angle of origin was usually orthogonal (58/60), but the coronary arteries arose tangentially, and passed intramurally, before emerging from the aorta in two hearts. In both cases, the origin was at or above the sinutubular junction.

\section{Distribution and branching pattern of coronary} circulation

All but two specimens (96\%) showed a dominant right pattern. In one heart with a dominant left pattern, the left coronary artery arose from the right hand sinus and the right coronary artery from the left hand sinus, with a side by side orientation of the pulmonary trunk and aorta.

The patterns of origin, and epicardial course relative to the great arteries, is summarised in table 1. The circumflex artery was absent in four specimens with an otherwise usual coronary pattern. In two cases there were dual left anterior descending coronary arteries.

\section{Origin of sinus node artery}

Seventy seven specimens were suitable for examination. The nodal artery arose from the coronary artery arising from the right hand sinus in $74(96 \%)$; the right coronary artery in 70 , the left coronary artery in two, and the circumflex coronary artery in two specimens. In two further cases, it arose from the right coronary artery arising from the left hand sinus. In these hearts, the nodal artery arose distant (20 $\mathrm{mm}$ and $37 \mathrm{~mm}$ ) to the origin of the right coronary artery, and coursed leftwards to the aorta. In the last case, it arose from the left coronary artery arising from the left hand sinus. In the other hearts, the nodal artery arose at a mean distance of $5.9 \mathrm{~mm}$ from the coronary arterial origin, but varied from $0-37 \mathrm{~mm}$. In eight hearts it arose at the origin of the right coronary artery.

\section{SURGICAL SERIES}

Coronary artery origins

The origins and course of the coronary arteries were described in 189 patients. There was an orifice in or above the left hand sinus (sinus 1) in 185 patients, and the coronary arteries arose within the sinus in 181 cases (98\%). In three cases where the coronary arteries had a high take off, there was an intramural course, but in one case it arose high above the sinutubular junction and descended onto the surface of the aorta before emerging to run round the front of the pulmonary trunk.

The radial origin was close to the centre of the sinus in 181 cases (98\%), being paracommissural in four. In three cases the coronary artery was intramural and, in one case, it arose high above the sinutubular junction above the commissure but was not intramural.

The angle of origin was orthogonal to the wall of the aorta in 179 cases (97\%). There were two patients with tangential origin, which were not intramural, and there were four with an intramural course of the main stem of the left coronary artery. In two of these cases, the 
left coronary artery arose above the commissure between the facing sinuses. In the third patient, the orifices of the left and right coronary arteries were located above the sinutubular junction, and very near to the commissure, being side by side and almost touching each other. Both had an initial intramural course. In the fourth patient there was a single left coronary artery which gave rise to the anterior descending, circumflex, and intramural right coronary arteries.

A coronary artery arose from the right hand sinus in 185 patients. It arose within the sinus in 183 patients (99\%), with high take off of the right coronary artery in two cases (one having single origin for left and right coronary arteries, and one with high and paracommissural origin of both, within millimetres of each other, both being intramural).

The radial origin was close to the centre of the sinus in 182 (98\%) patients, with three having a paracommissural origin. Two of the cases are those described above with high take off. The third had an artery arising close to the commissure between the right hand sinus (sinus 2) and the non-facing sinus.

The angle of origin was orthogonal in 181 $(98 \%)$ patients. No tangential origins were observed, but there were four with an intramural origin, three of them being a left coronary artery. In two patients a single orifice in sinus 2 gave rise to both right and left coronary arteries. The orifice itself was high and paracommissural. In the third patient, the left coronary artery arose from the right hand sinus and passed between aorta and pulmonary trunk, having an intramural segment. In this last case there was an accessory anterior descending coronary artery arising from the right hand sinus, which was found at surgery to have an intramural course.

Branching pattern of the coronary arteries A summary of the branching pattern is given in table 1 , with a similar range to that seen in the literature. The mortality is also given for each pattern. Four children had dual left anterior descending coronary arteries. In three cases this had not been detected preoperatively by echocardiography.

\section{Postoperative ventricular ischaemia}

The mortality of the arterial switch operation in this series was 20/176 (8/84 (9.5\%) at Guy's and St Thomas' Hospital, and 12/92 (13\%) at the Royal Brompton Hospital). Of those seven children with intramural coronary arteries, four died postoperatively with ventricular ischaemia and 11 others died with ischaemic complications. Of these, seven had unusual branching patterns. In these children, two had origins which were paracommissural, and two had high take off. The other four deaths occurred early in the surgical series, with no particular risk factors. A $\chi^{2}$ test confirms that all patterns were associated with a higher risk of death than the most usual pattern (1: left circumflex, and 2: right), $(\mathrm{p}<0.0001)$.

\section{Discussion}

ORIGINS OF CORONARY ARTERIES

We have shown that, in $20 \%$ of hearts studied at necropsy, one or both coronary arteries had high take off from the aortic root. This was divided equally between the arteries related to the facing sinuses (13\% each). No coronary arteries in our series arose from lower than $70 \%$ of the depth of the sinus. Paracommissural origin is also described, being more likely when arising from the contralateral facing sinus, and also more likely with high take off. Intramural coronary arteries were seen in a small number, but arteries with tangential take off were also seen. Tangential origin was more likely with paracommissural or high take off. In at least one case, the combination would have presented a difficult problem for the surgeon had an attempt been made to translocate the coronary arteries during an arterial switch operation.

It is important to place these results in the context of surgical repair. The location of coronary artery orifices relative to the aortic sinuses has been extensively studied in normal hearts, ${ }^{11}{ }^{15} 16$ and also in tetralogy of Fallot. ${ }^{17}$ In tetralogy of Fallot, the origin of the left coronary artery is more posterior, perhaps because of the abnormal development which produces a more rightward position of the aorta. In TGA, also usually seen with rightward aorta, this feature is of far more than academic interest, because of the need to translocate coronary arteries during the arterial switch operation. Although the patterns of branching have been extensively recorded, ${ }^{11-13}{ }^{18-20}$ little attention has been paid to the details of the sinusal origin. Although the relation of the aorta relative to the pulmonary trunk is more variable when the ventriculo-arterial connections are discordant as opposed to concordant, this factor does not seem to influence greatly the horizontal location of the orifices. As a greater proportion of orifices are located within the sinuses in TGA (87\% for the left and right hand sinuses) in comparison with the normal hearts $(69 \%$ and $78 \%$ for the left and right facing sinuses, respectively), and are also located deeper in the sinuses compared to the normal hearts $(p<0.001)$, perhaps because the aortic valve is at a higher position than the pulmonary valve, this provides an advantage when transferring the coronary arteries during the arterial switch operation.

In the landmark study of Angelini in otherwise normal hearts, three types of coronary artery orientation were identified relative to the sinus itself: orthogonal to the sinusal wall, tangential to the wall, and intramural. ${ }^{11}$ These patterns were also identified in our specimens. When the orifice was positioned at the centre of the sinus, the orientation of the proximal arterial segment was usually $(80 \%)$ orthogonal to the aortic wall. This orientation is ideal for the translocation of the coronary arteries. ${ }^{21}$ In the remaining $20 \%$ of specimens with high take off or paracommissural orifices, there was an acute angle of origin. Here, flow may be impaired following the coronary transfer. Such 
paracommissural origins are known to be a risk factor for the arterial switch operation. ${ }^{3-6}$

An intramural segment usually involves an anterior descending or left coronary artery arising from an orifice in the right hand sinus, or one which is just paracommissural. ${ }^{7-10}{ }^{20}$ This arrangement was found in three of our patients. The other patterns of intramural course seen in our study are known to represent significant risk factors for the arterial switch, and carried a high mortality (4/7 cases, $57 \%$ ). The incidence of intramural segments has been reported as $3-5 \%,{ }^{7-10}$ similar to that seen in our study. We also encountered a solitary artery with a stenotic orifice, as reported by Asou and colleagues. ${ }^{8}$

Intramural origin has been reported sporadically in normal subjects, ${ }^{22-25}$ especially when associated with subsequent course between the arterial trunks. These patterns are known to be associated with sudden death resulting from myocardial ischaemia, especially during exercise..$^{22-25}$ It is, therefore, important that transfer, relocation, and enlargement of the orifices, if needed, is performed during the arterial switch operation, allowing good outcome. ${ }^{8}$ We have confirmed that echocardiography is a useful technique for defining an intramural segment of a coronary artery. ${ }^{10}$ The presence of high take off or paracommissural origin is highly suggestive of an intramural coronary artery. Using these criteria, none of our patients with this abnormality were unrecognised preoperatively.

\section{CORONARY CIRCULATION}

The incidence of dominant right coronary arteries $(99 \%)$ in TGA is higher than in the normal population $(90 \%)^{26}{ }^{27}$ and in tetralogy of Fallot $(72 \%),{ }^{17}$ although the reason for this is not clear. Only two specimens showed a dominant left pattern, and in both the artery arose from the right hand sinus. The absence of the circumflex artery in $6 \%$ of our specimens might be an associated feature. All but three $(97 \%)$ of the arteries supplying the sinus node arose from the right hand sinus. Of these, 93\% took origin from the right coronary artery, a much greater proportion than in normal hearts. ${ }^{15}$ In two specimens the nodal artery arose distant from the origin of the right coronary artery. This might have involved stretching during surgical transfer. In $56 \%$ of the specimens, the origin of the nodal artery was near $(\leqslant 5 \mathrm{~mm})$ to the origin of its parent coronary artery, and was at the origin in $13 \%$. Such proximal origin may also lead to difficulty in surgical transfer, leading to potential kinking with loss of sinus rhythm postoperatively. ${ }^{5}$

CORONARY ARTERIAL BRANCHING PATTERNS

Embryological studies have shown that the coronary arteries develop after aortopulmonary septation, and that the distal major coronary arteries develop subsequent to formation of the arterial orifices - that is, by ingrowth to the sinuses ${ }^{1128}$ rather than outgrowth from the aortic wall. Thus, the coronary artery pierces the nearest site of the aortic sinus after the completion of aortopulmonary rotation. This satisfactorily explains the presence of intramural coronary arteries, since entry might be at a point above the sinutubular junction. The path of the artery is then likely to be within the wall of the aorta.

Many studies have tried to classify the branching patterns in numeric or alphabetic fashion. ${ }^{413} 14$ These categories lead only to confusion and disagreement. In addition a recent new system of classification (de Leval, personal communication, 1998) suggests a final group of cases which are all unclassifiable. In clinical practice, we suggest it is better to describe the patterns and thus avoid the potential confusion. Several coronary patterns were identified in our specimens and patients, all of which have been described before, and on which we shall not dwell. We suggest, therefore, that the coronary arteries should be described in a continuum based on their sinus of origin, and their radial origin, vertical origin, and angle of origin.

Dual anterior descending coronary arteries were a notable feature in six cases, nonetheless, and deserve attention since they provided a trap for the unwary echocardiographer. On three occasions, the surgeon was surprised, but in no case was there technical difficulty in transfer. Identification of one anterior descending artery, therefore, does not preclude the existence of another.

\section{CONCLUSION}

We have shown that some children with abnormal coronary arteries died with ventricular ischaemia while undergoing the arterial switch operation, some without intramural coronary arteries, the anomalies being detected by echocardiography. Subsequent to our clinical and morphological results, we hope it will be easier to identify those patients with an increased risk of surgery. The choice for each surgical unit lies between referral to a low risk institution, requesting surgical advice in the "home" institution during the arterial switch procedure, or performing an alternative operation. The latter seems the worst option, since it does not remove the subsequent known risk of sudden death.

We are grateful to our clinical colleagues at Guy's Hospital and the Royal Brompton Hospital for permission to report findings from patients in their care. Support is acknowledged from the Joseph Levy Foundation and the British Heart Foundation.

1 Kirklin JW, Blackstone EH, Tchervenkov CI, et al, for the Congenital Heart Surgeons Society. Clinical outcomes Congenital Heart Surgeons Society. Clinical outcomes after the arterial switch operation for transposition. Patient, support, procedural, and tion 1992;86:1501-15.

2 Mayer JE, Sanders SP, Jonas RA, et al. Coronary artery pattern and outcome of arterial switch operation for transposition of the great arteries. Circulation 1990;82(suppl IV):IV139-45

3 Planche C, Bruniaux J, Lacour-Gayet F, et al. Switch operation for transposition of the great arteries in neonates. A study of 120 patients. F Thorac Cardiovasc Surg 1988;96: 353-63.

4 Yacoub MH, Radley-Smith R. Anatomy of the coronary arteries in transposition of the great arteries and methods for their transfer in anatomical correction. Thorax 1978;33: 418-24.

5 Yamaguchi M, Hosokawa Y, Imai Y, et al. Early and midterm results of the arterial switch operation for transposition of results of the arterial switch operation for transposition of
the great arteries in Japan. $\mathcal{F}$ Thorac Cardiovasc Surg 1990; 100:261-9.

6 Day RW, Laks H, Drinkwater DC. The influence of coronary anatomy on the arterial switch operation in neonates. $\mathcal{F}$ Thorac Cardiovasc Surg 1992;104:706-12. 
7 Sim EKW, van Son JAM, Julsrud PR, et al. Aortic intramural course of the left coronary artery in dextrotransposition
of the great arteries. Ann Thorac Surg 1994;57:458-60.

8 Asou T, Karl TR, Pawade A, et al. Arterial switch: translocation of the intramural coronary artery. Ann Thorac Surg tion of the intram

9 Gittenbergerde Groot AC, Sauer U, Quaegebeur J. Aortic intramural coronary artery in three hearts with transposition of the great arteries. $\mathcal{F}$ Thorac Cardiovasc Surg 1986;91 566-71.

10 Pasquini L, Parness IA, Colan SD, et al. Diagnosis of intramural coronary artery in transposition of the great arteries using twodimensional echocardiography. Circulation 1993; 88:1136-41.

11 Angelini P. Normal and anomalous coronary arteries: definition and classification. Am Heart f 1989;117:418-28.

12 Gittenbergerde Groot AC, Sauer U, Oppenheimer D, et al. Coronary arterial anatomy in transposition of the great Coronary arterial anatomy in transposition of the great
arteries: a morphologic study. Pediatr Cardiol 1983;4 (suppl 1): 1524 .

13 Amato JJ, Zelen J, Bushong J. Coronary arterial patterns in complete transposition classification in relation to the arterial switch procedure. Cardiol Young 1994;4:329-39.

14 Chiu IS, Chu SH, Wang JK, et al. Evolution of coronary artery pattern according to short-axis aortopulmonary rotation: a new categorization for complete transposition of the great arteries. F Am Coll Cardiol 1995;26:250-8.

15 McAlpine WA. The coronary arteries. In: McAlpine WA, ed. Heart and coronary arteries. Berlin: SpringerVerlag, 1979:140-201.

16 Muriago M, Sheppard MN, Ho SY, et al. The location of the coronary arterial orifices in the normal heart. Clin Anat 1997;10:16.

$17 \mathrm{Li} \mathrm{J}$, Soukias ND, Carvalho JS, et al. Coronary arterial anatomy in tetralogy of Fallot: morphological and clinical correlations. Heart 1998;80:174-83.
18 Sim EKW, van Son JAM, Edwards WD, et al Coronary artery anatomy in complete transposition of the great artery anatomy in complete transposition
arteries. Ann Thorac Surg 1994;57:890-4.

19 Pasquini L, Sanders SP, Parness IA, et al. Coronary echocardiography in 406 patients with d-loop transposition of the great arteries. $\mathcal{F}$ Am Coll Cardiol 1994;24:763-8.

20 Sim EKW, Julsrud PR, van Son JAM, et al. Preoperative diagnosis of coronary artery anatomy in dextrotransposition of the great arteries. Mayo Clin Proc 1994;69:2832.

21 Yacoub MH. Anatomic correction of transposition of the great arteries at the arterial level. In: Sabiston DC, Spencer FC, eds. Surgery of the chest. Philadelphia: WB Saunders, FC, eds. Surgery

22 Mustafa I, Gula G, Radley-Smith R, et al. Anomalous origin of the left coronary artery from the anterior aortic sinus: a potential cause of sudden death. F Thorac Cardiovasc Surg 1981;82:297-300.

23 Benson PA. Anomalous aortic origin of the coronary artery with sudden death: case report and review. Am Heart 7 1970;79:254-7.

24 Cohen LS, Shaw LD. Fatal myocardial infarction in an 11 year old boy associated with unique coronary artery anomaly. Am f Cardiol 1967:19:420-3.

25 Cheitlin MD, De Castro CM, McAllister HA. Sudden death as a complication of left coronary origin from the anterior sinus of Valsalva. Circulation 1974;50:780-6.

26 Helfant RH, Banka VS. A clinical and angiographic approach to coronary heart disease. Philadelphia: FA Davis Co, 1978:23.

27 Gensini GG. Coronary arteriography. Mount Kisco, New York: Futura, 1975:168.

28 Bogers AJ, Gittenberger-de Groot AC, Poelmann RE, et al. Development of the origin of the coronary arteries, a matter of ingrowth or outgrowth? Anat Embryol (Berl) 1989;180:437-41. 